\title{
OBSERVATIONS OF COSMIC MASERS
}

\author{
P.J.Diamond \\ National Radio Astronomy Observatory \\ Edgemont Road \\ Charlottesville VA 22903-2475
}

ABSTRACT .

A review is presented of recent results from interferometric observations of cosmic masers. Several topics will be discussed, including MERLIN/VLA/VLBI observations of megamasers; recent results on masers in star forming regions and around late-type stars and some new results on the effects of interstellar scattering on maser spot sizes.

\section{INTRODUCTION}

Astronomical masers were discovered in 1965 (Weaver et al.), but although they have been studied extensively in the intervening 22 years very little has been uncovered concerning the physics of their emission, or the conditions that give rise to it. Indeed, the study of masers per se reveals almost no information concerning the physical conditions in their immediate environment. This was summarized in a comment I once overheard in the corridors of a well-known European observatory : "Why observe masers, the damn things tell you nothing!". However this is not, of course, strictly true; observations of masers can provide detailed information on the dynamics of a region, be it a circumstellar shell, an outflow near an HII region or the central parts of a galaxy; they can also provide accurate estimates of the distance to the maser sources through observations of the proper motions of $\mathrm{H}_{2} \mathrm{O}$ masers, or the method of phase-lags (Schultz et al. 1978); the circular polarization observed in $\mathrm{OH}$ and SiO masers can be used to determine the magnetic field strengths in the immediate vicinity of the masers; observation of the sizes of the masers provide extremely useful information on the distribution of scattering material in our galaxy. These are some of the many reasons why we observe masers and I shall discuss some of the more recent results obtained. 


\section{EXTRAGALACTIC MASERS}

Extragalactic masers were first discovered in 1973 in the galaxy NGC253 (Whiteoak and Gardner 1973). The OH masers observed, although one to two orders of magnitude stronger than Galactic masers, were not particularly accesible due to their large distance and thus low flux density. In 1982 a different class of extragalactic $\mathrm{OH}$ maser was discovered in the peculiar galaxy IC4553 (Baan et al. 1982). It was immediately dubbed a "megamaser" since its intrinsic strength was about $7 \times 10^{7}$ that of the $W 3(\mathrm{OH})$ masers in our Galaxy. Following the initial excitement of the discovery telescopes all over the world began searching for new megamasers until, at the time of writing, 15 are known (see Table 1).

In addition to $\mathrm{OH}$ two other molecules have been observed to be masing in other galaxies. In 1986 Baan et al. (1986) reported the detection of a powerful $\mathrm{H}_{2} \mathrm{CO}$ maser in IC4553 (and possibly one in NGC 3079). $\mathrm{H}_{2} \mathrm{O}$ masers were detected in external galaxies as long as 10 years ago (Churchwell et al. 1977; Lepine and Dos Santos 1977; Huchtmeier et al. 1978, 1980; Scalise and Braz, 1981). However these sources are comparable in luminosity to the strongest Galactic masers $(\leq 1 \mathrm{~L} \odot)$. Much more luminous $\mathrm{H}_{2} \mathrm{O}$ masers have since been found and to date $6 \mathrm{H}_{2} \mathrm{O}$ megamasers are known (see Table 2).

TABLE 1

Known OH Megamaser Galaxies

\begin{tabular}{ll}
\hline \hline \multicolumn{1}{c}{ Galaxy } & \multicolumn{1}{c}{ References } \\
\hline IC4553 & Baan, Wood, and Haschick 1982 \\
NGC 3690 & Baan 1985 \\
Mrk 231 & Baan 1985; Kázes and Dickey 1985 \\
Mrk 273 & Baan, Haschick and Schmelz 1985 \\
& Bottinelli et al. 1985 \\
IIIZW 35 & Chapman et al. 1986 \\
IRAS 11506-3851 & Norris et al. 1986 \\
Arp 148 & Bottinelli et al. 1986 \\
IIZW 96 & Bottinelli et al. 1986 \\
& Baan, Henkel and Haschick 1987 \\
IRAS 17208-0014 & Bottinelli et al. 1986 \\
ZW15107+0724 & Baan, Henkel and Haschick 1987 \\
IRAS 16399-0937 & Staveley-Smith et al. 1986 \\
ZW475.056 & Mirabel and Sanders 1987 \\
IRAS 1017+08 & Mirabel and Sanders 1987 \\
IRAS 1211+03 & Kázes et al. 1987 \\
NGC 4418 & Bottinelli et al. 1987 \\
\hline
\end{tabular}


TABLE 2

Known $\mathrm{H}_{2} \mathrm{O}$ Megamaser GalaXies

\begin{tabular}{ll}
\hline \hline Galaxy & \multicolumn{1}{c}{ References } \\
\hline NGC 4945 & Dos Santos and Lepine 1979 \\
Circinus & Gardner and Whiteoak 1982 \\
NGC 1068 & Claussen et al. 1984 \\
NGC 4258 & Claussen et al. 1984 \\
NGC 3079 & Henkel et al. 1984 \\
IC 10 & Henkel et al. 1986 \\
\hline
\end{tabular}

The extragalactic masers so far discovered are all believed to be associated in some way with regions of star formation, whether they are physically associated with $O B$ stars as might possibly be the case for extragalactic $\mathrm{H}_{2} \mathrm{O}$ masers, or whether their emission is indirectly triggered by a burst of star formation. However Wood et al. (1986) and Whiteoak et al. (priv. comm.) have recently discovered several $1612 \mathrm{MHz} \mathrm{OH}$ masers associated with OH/IR stars in the Large Magellanic Cloud. These masers appear to be weaker than their Galactic counterparts but show otherwise similar characteristics.

Interferometric observations of extraglactic masers

IC4553 is the only extragalactic OH megamaser whose structure is at all well determined. Baan and Haschick (1984) used the VLA to map the spatial distribution of the $\mathrm{OH}$ emission. The source was only slightly resolved but two interesting facts emerged from the data; a) the nuclear continuum source and $\mathrm{OH}$ emission region appeared to be perfectly superposed, and b) the velocity structure of the $\mathrm{OH}$ emission was consistent with solid body rotation of a molecular disc. Norris et al. (1985) used MERLIN to produce much higher resolution maps of the $\mathrm{OH}$ and $18 \mathrm{~cm}$ continuum emission. They found the nucleus to be a core-dominated triple similar to those seen in other active galactic nuclei and the $\mathrm{OH}$ masers appeared to amplify the triple structure (it now appears that the triple structure observed was caused by the symmetrization propeties of self-cal and that the true structure is a double, this does not however change any of the results). The $\mathrm{OH}$ emission from IC4553 has also been detected with VLBI (Diamond et al. 1987), the VLB image is seen to be a double, and the eastern component appears to breakup into a number of smaller masers. No continnuum emission was detected with VLBI. Four other $\mathrm{OH}$ megamasers have been observed with the VLA; Mrk 273 (Schmelz 1987); IIIZw35 (Chapman priv. comm. ); IRAS 17208-0014 (Martin priv. comm.) and IRAS 11506-3851 (Norris priv. comm. ). As expected the OH emission from each is superposed on the continuum emission but none of the sources were resolved.

Interferometric observations of extragalactic $\mathrm{H}_{2} \mathrm{O}$ masers have been conducted recently by Claussen and Lo (1986) and Haschick 
et al. (1987). These observations have revealed the surprising result that the extragalactic $\mathrm{H}_{2} \mathrm{O}$ masers in NGC 4258, 1068 and 3079 are confined to very small regions $(\leq 4 \mathrm{pc}$ ) and appear to be coincident with compact sources of radio emission in the nuclei of the galaxies. In addition the masers have luminosities $\geq 120 \mathrm{~L} \odot(\sim 500 \mathrm{~L} \odot$ for NGC3079), two orders of magnitude more powerful than the Galactic $\mathrm{H}_{2} \mathrm{O}$ masers in W49. It is evident that standard pumping schemes (which have difficulty explaining the Galactic $\mathrm{H}_{2} \mathrm{O}$ masers) cannot explain the extragalactic masers. Strelnitskij (1984) and Kylafis and Norman (1986) have proposed collisional pumping schemes involving hydrogen and electrons at different kinetic temperatures which may be able to explain these powerful masers since collisional quenching of the pump does not occur, thereby giving rise to more powerful masers.

\section{A Model for Extragalactic Megamasers}

Due to the relative paucity of observational data on extragalactic masers no detailed model explaining them has yet emerged. However some common links are evident. First, almost all megamasers appear to be superposed on continuum emission originating in the host galaxy, this would imply (at least in the case of $\mathrm{OH}$ megamasers) that the maser is a result of low gain amplification of the continuum by excited foreground molecular gas. Secondly, the galaxies with megamaser emission are usually very strong infrared sources, the observed IR emission is quite sufficient to excite the molecular gas containing the masers. Baan et al. (1987) have demonstrated that for the $\mathrm{OH}$ megamasers $L_{O H}$ is proportional to $L_{I R}^{2}$. It is suggested that the cause of the continuum emission and high IR luminosity is that the megamaser galaxies recently underwent, or are undergoing, a burst of star formation. A third link common to the $\mathrm{OH}$ megamaser galaxies is that many (if not all) of them appear to have an edge-on molecular disc, this increases the probability of seeing inverted molecular clouds along the line of sight.

Baan et al. 1987 suggest that the $\mathrm{OH}$ megamaser galaxies are a very early stage of Seyfert 2 or HII nuclear activity in a dusty galaxy and that possibly all Seyfert and HII nucleii exhibited maser activity when they turned on.

The $\mathrm{H}_{2} \mathrm{O}$ megamaser galaxies tend to have slightly different properties than the $\mathrm{OH}$ emitting galaxies. Their IR luminosities are less and the compact continuum sources also tend to be weaker, although the masers do coincide with continuum sources. Haschick et al. (1987) suggest that the coincidence of the masers and continuum sources may be causally related due to either a) the $\mathrm{H}_{2} \mathrm{O}$ masers may be amplifying the background radiation; or b) the radio source is an indication of a massive cluster of $O B$ stars that may be providing the maser pump power (although see above). 


\section{INTERSTELLAR MASERS}

Interferometric observations of Galactic interstellar masers is not a subject which has been pursued with any great vigour over the last few years, however a few results do stand out. The proper motion studies of $\mathrm{H}_{2} \mathrm{O}$ masers spots in HII regions have produced accurate distances to several sources; Genzel et al. (1981a) have determined a distance of $480 \pm 80$ pc for Orion-KL; Genzel et al. (1981b) and Schneps et al. (1981) determined the distance to the $W 51$ molecular cloud to be $7 \pm 1.5 \mathrm{kpc}$; more recently Reid et al. (1987) have determined the distence to Sgr-B2 to be $7.1 \pm 1.5 \mathrm{kpc}$ implying that the Galactic centre is significantly closer than the previous standard value of $10 \mathrm{kpc}$.

OH masers are also associated with regions of star formation and can provide the observer with a wealth of information on the dynamics of such objects. Gaume and Mutel (1987) have observed 11 star forming regions with the VLA and, for the first time, are able to produce some statistics concerning the relationship of $\mathrm{OH}$ masers and HII regions. They find that all four ground state $\mathrm{OH}$ transitions are observed but that the mainline transitions (at 1665 and $1667 \mathrm{MHz}$ ) are usually the strongest. They also observed that the different maser transitions are frequently found very close to each other ( $\leq 0.5$ ") suggesting that several transitions can be pumped together. Regarding the positional relationship between $\mathrm{OH}$ masers and the $15 \mathrm{GHz}$ structure of the HII regions they find that the overall distribution of maser features is strongly peaked near the edge of the continuum sources, and that in those HII regions which have a cometary-like structure (suggesting motion between the HII region and the surrounding neutral material) the strongest $\mathrm{OH}$ masers are projected onto the upstream leading edge of the HII regions.

Baart and Cohen (1985) and Baart et al. (1986) have used MERLIN to observe the $\mathrm{OH}$ masers in $\mathrm{G} 45.1+01$ and $W 75 \mathrm{~N}$, both active regions of star formation. In both sources they find that the $\mathrm{OH}$ masers lie near the edges of the HII regions, similar to the result obtained by Gaume and Mutel (1987); they also suggest that there is a tendency for $\mathrm{OH}$ masers to lie at greater distances from more luminous stars. There is some discussion as to the nature of the OH/HII regions; Reid et al. (1980), in their definitive study of the $\mathrm{OH}$ masers in W3 $(\mathrm{OH})$, suggested that the masers were part of a remnant-accreting envelope which is still collapsing towards a newly formed star. This conclusion was arrived at since many of the masers detected by Reid et al. (1980) were projected against, and appeared to be redshifted with respect to, the underlying HII region. However more sensitive observations of the $W 3(\mathrm{OH})$ masers by Norris et al. (1982), and observations of other HII region masers by Baart and Cohen (1985) and Gaume and Mutel (1987) suggest that in general OH masers are expanding away from their parent HII regions.

W3 $(\mathrm{OH})$ is probably the best studied of all OH/HII region masers sources and observations have continued with recent VLBI observations 
of excited state $\left({ }^{2} \pi_{1 / 2}, J=1 / 2, \Delta F=1-0\right)$ OH masers at a frequency of $4765 \mathrm{MHz}$ by Baudry et al. (1987). The VLBI data implied apparent brightness temperatures $\geq 9 \times 10^{8} \mathrm{~K}$, and some spatial and velocity correlation of the $4765 \mathrm{MHz}$ masers with the $1665 / 7 \mathrm{MHz}$ and $6035 \mathrm{MHz}$ masers is observed.

Another strong masing molecule seen in W3(OH) is $\mathrm{CH}_{3} \mathrm{OH}$. There are several transitions detected in various sources with the $2_{0} \rightarrow 3_{-1} E$ transition recently observed to have fluxes between $100-1200 \mathrm{Jy}$ in some sources (Batrla et al. 1987; Norris et al. 1987), but at the relatively inaccesible frequency (for most interferometers) of $12 \mathrm{GHz}$. However the $9_{2} \rightarrow 10_{1} A^{+}$transition at $23 \mathrm{GHz}$ is observed in $\mathrm{W} 3(\mathrm{OH})$ at a flux density of $\sim 10 \mathrm{Jy}$. This transition was recently observed with both the VLA (Menten priv. comm.) and VLBI (Johnston priv. comm.). The VLBI experiment was unable to detect any line fringes implying spot sizes of between 5 and 40 mas, but the lack of VLBI fringes may be due to instrumental problems. The image produced with the VLA data is a great surprise since the $\mathrm{CH}_{3} \mathrm{OH}$ masers are seen to correlate almost exactly with the gross spatial and velocity structure of the 1665 OH masers.

\section{STELLAR MASERS}

Molecular masers are also associated with oxygen-rich M-type giant and supergiant stars. Strong $\mathrm{OH}, \mathrm{H}_{2} \mathrm{O}$ and $\mathrm{SiO}$ masers are observed in such objects and it is now commonly accepted that the masers lie in an expanding circumstellar shell. Since the masing transitions of the three molecular maser species are excited at very different temperatures it is assumed that they lie at increasing distances from the stellar surface with the Sio being excited close to the star and the $\mathrm{OH}$ at typical distances $\geq 10^{16} \mathrm{~cm}$ from the star. The envelopes typically have expansion velocities of $5 \rightarrow 40 \mathrm{~km} \mathrm{~s}^{-1}$ and mass loss rates of $\sim 10^{-6} \rightarrow 5 \times 10^{-4} M \odot \mathrm{yr}^{-1}$. In addition to the masers in oxygenrich stars Guilloteau et al. (1987) have recently discovered a strong maser ( $75 \mathrm{Jy}$ ) from a vibrationally excited transition of $\mathrm{HCN}$ in the envelope of the carbon-rich star CIT 6 .

Thers is now a large database of interferometric observations of stellar masers. Booth et al. (1981) first demonstrated convincingly that $\mathrm{OH}$ masers lay in an expanding, spherical envelope with observations of the $1612 \mathrm{MHz} \mathrm{OH}$ masers in $\mathrm{OH} 127.8-0.0$. This model has since been confirmed for many sources by VLA and MERLIN observations (e.g. Bowers et al. 1983; Diamond et al. 1985a and references therein). Models of maser emission predict (as mentioned above) that the different molecular masers should lie at different distances from the central exciting star; it is only in the last year or so that detailed observations of the different molecular species have become available (Chapman and Cohen, 1986; Diamond et al. 1985b; Diamond et al. 1987) that 
show the models to be correct in their overall details.

VLBI observations of stellar OH masers have been useful to determine the relative locations and sizes of individual masers spots but, until recently, had only been performed on a few sources since it had been assumed that in general stellar masers were larger than the equivalent interstellar masers. However recent VLBI observations of $\mathrm{OH}$ masers have revealed the presence of very compact emission spots (Norris et al. 1984; Sivagnanam et al. 1987) in both distant OH/IR stars and Mira Variables. Such observations are important since it appears the masers are amplifying the thermal emission from the star and thus lie directly along the line of sight to it implying that with both radio and optical astrometric observations these sources could be used to relate the FK4 optical reference frame with the radio reference frame (see de Vegt et al. 1986).

Unlike interstellar $\mathrm{OH}$ masers, most stellar sources exhibit little or no polarization. There are some very notable exceptions such as the supergiant stars and the variables $W$ Hya and $U$ Ori. It is assumed that the Zeeman effect is responsible for the observed polarization and, given that, it should be possible to determine the magnetic field strength in the immediate vicinity of the masers and thus extapolate back to determine the field strength at the stellar surface. Early experiments (Reid et al. 1979) estimated the magnetic field at the surface of $U$ Ori to be $\sim 10 \mathrm{G}$ and at the surface of IRC +10420 to be $\sim 100 \mathrm{G}$. Recently Barvainis et al. (in prep.) have observed significant circular polarization in SiO masers associated with late-type stars which imply magnetic fields in the immediate vicinity of the masers $\sim 10 \rightarrow 100 \mathrm{G}$, this is a much more direct measurement of the magnetic field. McIntosh (priv. comm.) has made VLBI maps of the SiO maser distribution in the envelopes of two latetype stars which he combined with single-dish polarimetric data to produce maps which may show the pattern of the magnetic field lines around the stars.

\section{INTERSTELLAR SCATTERING}

Previous VLBI observations of the spot sizes of masers (Bowers et al. 1980) suggested that there was a tendency for more distant masers to be larger. Since it is unlikely that this is an intrisic property of distant masers it was assumed that the spot sizes of these masers were broadened by scattering by intervening diffuse HII regions. To date very little in the way of observational data of the distribution of maser spot sizes within the Galaxy has been gathered, howvever Kemball et al. (1987) have observed $17 \mathrm{OH}$ maser sources in the galactic plane from longitude $355^{\circ}$ to $50^{\circ}$, they only detect sources at longitude $\geq 35^{\circ}$ implying that interstellar scattering increases at low Galactic longitudes. 


\section{ACKNOWLEDGEMENTS}

I would like to thank Willem Baan, Jessica Chapman, Jim Cohen, Aubrey Haschick, Christian Henkel, Gordon Mcintosh, Ray Norris, John Whiteoak and Tom Wilson who willingly and quickly provided me with reams of preprints and yet to be published data without which this review could not have been written.

\section{REFERENCES}

Baan, W. A. 1985, Nature, , 315, 26.

Baan, W. A. and Haschick, A. D., 1984, Ap. J., 279, 541.

Baan, W. A., Wood, P. A. D. and Haschick, A. D., 1982 Ap. J.(Letters), , 260, L49.

Baan, W. A., Haschick, A. D. and Schmelz, J. T., 1985 Ap. J.(Letters),, 298, L51.

Baan, W. A., Güsten, R. and Haschick, A. D., 1986, Ap.J., , 305, 830 .

Baan, W. A., Henkel, C. and Haschick, A. D., 1987, Ap. J., , in press.

Baart, E. E. and Cohen, R. J., 1985, M.N.R.A.S., , 213, 641 .

Baart, E. E., Cohen, R. J., Davies, R. D., Norris, R. P. and Rowland, P. R., 1986, M.N.R.A.S., 219, 145.

Barvainis, R., McIntosh, G. and Predmore, C. R., 1987, preprint.

Batrla, W., Matthews, H. E., Menten, K. M. and Walmsley, C. M., 1987 , Nature, $326,49$.

Baudry, A., Diamond, P. J., Graham, D. A., Walmsley, C. M., Guilloteau, S. and Booth, R. S., 1987, in preparation.

Booth, R. S., Kus, A. J., Norris, R. P. and Porter, N. D., 1981, Nature, 291,382 .

Bottinelli, L., Fraix-Burnet, D., Gougenheim, L., Kázes, I., Le Squeren, A. M., Patey, I., Rickard, L. J. and Turner, B. E., 1985 Astr. Ap. (Letters), , 151, L7.

Bottinelli, L., Dennefeld, M., Gougenheim, L., Le Squeren, A. M., Martin, J. M. and Paturel, G., 1986, Activity in Stars and Galaxies, in press.

Bottinelli, L., Gougenheim, L., Le Squeren, A. M., Martin, J. M., Dennefeld, M. and Paturel, G., 1987, IAU Circ. 4379

Bowers, P. F., Johnston, K. J. and Spencer, J. H., 1983, Ap. J., , 274, 733.

Bowers, P. F., Reid, M. J., Johnston, K. J., Spencer, J. H. and Moran, J. M. , 1980, Ap. J., , 242, 1088.

Chapman, J. M. and Cohen, R. J., 1986, M.N.R.A.S., , 220, 513.

Chapman, J. M., Cohen, R. J., Pointon, L., Staveley-Smith, L. and Unger, S. W., 1986, IAU Circ. 4180

Churchwell, E., Witzel, A., Huchtmeier, W. K., Pauliny-Toth, I., Roland, J., Sieber, W., 1977, Astr. Ap., , 54, 969.

Claussen, M. J., Heiligman, G. M. and Lo, K. -Y., 1984, Nature, , 310, 298.

Claussen, M. J. and Lo, K. -Y., 1986, Ap. J., , 308, 592.

de Vegt, Ch., Kleine, Th., Johnston, K. J., Bowers, P. F. and Spencer, J. H., 1987 , preprint. 
Diamond, P. J., Norris, R. P., Rowland, P. R., Booth, R. S. and Nyman, L. -A., 1985a, M.N.R.A.S., 212, 1 .

Diamond, P. J., Norris, R. P. and Booth, R. S., 1985b, M.N.R.A.S., 216, $1 \mathrm{p}$.

Diamond, P. J., Johnston, K. J., Chapman, J. M., Lane, A. P., Bowers, P. F., Spencer, J. H. and Booth, R. S., 1987, Astr. Ap., , 174, 95.

Diamond, P. J., Norris, R. P., Baan, W. A. and Booth, R. S., 1987, in preparation.

Dos Santos, P. M. and Lepine, J. R. D., 1979, Nature, , 278, 34.

Gardner, F. F. and Whiteoak, J. B., 1982, M.N.R.A.S., 201, 13p.

Gaume, R. A. and Mutel, R. L., 1987, preprint.

Genzel, R., Reid, M. J., Moran, J. M. and Downes, D., 1981a, Ap.J., 244,884 .

Genzel, R., Downes, D., Schneps, M. H., Reid, M. J., Moran, J. M., Kogan, L. R., Kostenko, V. I., Matveyenko, L. I. and Rönang, B., 1981b, Ap. J., , 247, 1039.

Guilloteau, S., Omont, A. and Lucas, R., 1987, Astr. Ap. (Letters),, 176, L24.

Haschick, A. D., Baan, W. A., Schneps, M. H., Reid, M. J. and Moran, J. M. , 1987, in preparation.

Henkel, C., Gusten, R., Downes, D., Thum, C., Wilson, T. L. and Biermann, P., 1984, Astr. Ap. (Letters), 141, L1.

Henkel, C., Wouterloot, J. and Bally, B., 1986 Astr. Ap., , 155, 193.

Huchtmeier, W. K., Witzel, A., Kuhr, H., Pauliny-Toth, I. and Roland, J., 1978, Astr. Ap., , 64, L21.

Huchtmeier, W. K., Richter, O. -G., Witzel, A. and Pauliny-Toth, I., 1980, Astr. Ap., , 91, 259.

Kázes, I. and Dickey, J. M., 1985, Astr. Ap. (Letters), , 229, L91.

Kázes, I., Mirabel, I. F. and Sanders, D. B., 1987, IAU Circ. 4362.

Kemball, A., Diamond, P. J. and Mantovani, F., 1987, in preparation.

Kylafis, N. D. and Norman, C., 1986, Ap. J.(Letters), , 300, L73.

Lepine, J. R. D. and Dos Santos, P. M., 1977, Nature, , 270, 501.

Mirabel, I. F. and Sanders, D. B., 1987, preprint.

Norris, R. P., Booth, R. S. and Diamond, P. J., 1982, M.N.R.A.S., 201, 209.

Norris, R. P., Booth, R. S., Diamond, P. J., Nyman, L-A, Graham, D. A. and Matveyenko, L. I., 1984, M.N.R.A.S., , 208, 435.

Norris, R. P., Baan, W. A., Haschick, A. D., Diamond, P. J. and Booth, R. S., 1985, M.N.R.A.S., , 213, 821.

Norris, R. P., Whiteoak, J. B., Gardner, F. F., Allen, D. A. and Roche, P. F., 1986, M.N.R.A.S., , 221, 51p.

Norris, R. P., Caswell, J. L., Gardner, F. F. and Wellington, K. J., 1987, preprint.

Reid, M. J., Moran, J. M., Leach, R. W., Ball, J. A., Johnston, K. J., Spencer, J. H. and Swenson, G. W., 1979, Ap. J. (Letters), , 227, L89.

Reid, M. J., Haschick, A. D., Burke, B. F., Moran, J. M., Johnston, K. J. and Swenson, G. W., 1980, Ap. J., 239, 89.

Reid, M. J., Schneps, M. H., Moran, J. M., Gwinn, C. R., Genzel, R., Downes, D. and Rönang, B., 1987, IAU 115.

Scalise, E. and Braz, M. A., 1981, Nature, , 290, 36.

Schmelz, J. T., 1987, Ph.D Thesis 
Schneps, M. H., Lane, A. P., Downes, D., Moran, J. M., Genzel, R. and Reid, M. J., 1981, Ap. J., , 249, 124.

Schultz, G. V., Sherwood, W. A. and Winnberg, A., 1978, Astr. Ap. (Letters), , 63, L5.

Sivagnanam, P., Diamond, P. J., Ls Squeren, A. M., Daigne, G., Biraud, F. and Ortega-molina, A., 1987, in preparation.

Staveley-Smith, L., Unger, S. W., Cohen, R. J., Chapman, J. M. and Pointon, L., 1986, IAU Circ. 4248

Strelnitskij, V. S., 1984, M.N.R.A.S., $207,339$.

Weaver, H., Williams, D. R. W., Dieter, N. H. and Lum, W. T., 1965, Nature, , 208, 29.

Whiteoak, J. B. and Gardner, F. F., 1973, Ap. Letters, , 15, 211.

Wood, P. R., Bessell, M. S. and Whiteoak, J. B., 1986, Ap. J.(Letters),, 306, L81. 